\title{
Comparaison de la mortalité par cancer dans le Limousin et le Poitou-Charentes
}

\author{
(Etude préliminaire 1968-1975)
}

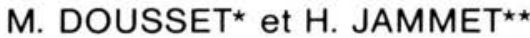 \\ (Manuscrit reçu le 8 novembre 1984)
}

\begin{abstract}
RÉSUME
Les deux régions Limousin et Poitou-Charentes sont géologiquement très différentes ; en moyenne, un habitant de la première région reçoit une dose supplémentaire de $65 \mathrm{mrem}$ par an (rayonnement tellurique) et la concentration du radon dans les habitations du Limousin est, en moyenne, trois à quatre fois plus élevée qu'en Poitou-Charentes.

Cette première étude confronte les taux comparatifs de mortalité par différents types de tumeurs malignes ( $n^{\circ} 20$ à 27 de la liste $D M$ de I'INSERM) pour les années allant du recensement de 1968 à celui de 1975 et pour tous àges confondus selon la population de référence 1968. Aucune différence significative n'apparaît pour ces différents types de cancers, sauf pour les cancers de l'appareil digestif pour lesquels une étude plus poussée s'impose. Le résultat le plus surprenant concerne les tumeurs malignes de l'appareil respiratoire pour lesquelles aucune différence, même minime, n'apparaît ; la consommation de tabac par habitant est pratiquement la même, en moyenne, pour les deux régions.
\end{abstract}

\section{ABSTRACT}

The geology of the Limousin and Poitou-Charentes regions is very different; on an average, in the Limousin region, an inhabitant receives a supplementary dose of 65 mrem per year (terrestrial radiation) and the indoor radon concentration is 3.4 times higher than in the Poitou-Charentes region.

The comparative rates of mortality for different types of malignant tumours ( $N \cdot 20-27$ of the INSERM DM list) were studied for the period extending from the 1968 to the 1975 census and for all ages according to the 1968 reference population. No significant difference appeared for these different types of cancers, except for cancers of the digestive system, which require a more thorough study. The more surprising result concerned malignant tumours of the respiratory system for which no difference - even small - appears ; tobacco consumption per inhabitant was practically the same, on an average, in both regions.

* Conseiller scientifique du Service central de protection contre les rayonnements ionisants, 7, rue de la Gruerie, 91190 Gif-sur-Yvette.

** Commissariat à l'énergie atomique, Institut de protection et de sûreté nucléaire, BP 6, 92260 Fontenay-aux-Roses. 
Les trois départements de la région du Limousin (Corrèze, Creuse et Haute-Vienne) présentent des caractéristiques géologiques très différentes de celles des départements qui constituent la région Poitou-Charentes (Charente, Charente-Maritime, Deux-Sèvres, Vienne).

Ces différences se traduisent par des niveaux moyens de radioactivité naturelle nettement plus élevés pour la première région que pour la seconde.

La mesure des doses dues aux rayonnements cosmiques et aux rayonnements $\gamma$ issus soit du sol, soit des matériaux de construction, a été effectuée dans les années passées [4] et des valeurs moyennes ont pu être obtenues à partir de 917 mesures dans le Limousin et 1345 mesures en PoitouCharentes ; ces mesures ont été faites à l'air libre et dans les habitations. Les moyennes s'élèvent à environ $145 \mathrm{mrem} / \mathrm{an}(1,45 \mathrm{mSv} / \mathrm{an})$ pour la première région et à $80 \mathrm{mrem} / \mathrm{an}(0,8 \mathrm{mSv} / \mathrm{an})$ pour la seconde.

Pour le radon, principalement dans les habitations, des campagnes de mesure sont en cours. Pour le Limousin, une première série de mesures [7] portant sur une centaine d'habitations faisait apparaître un niveau moyen de concentration en radon environ 5 fois plus élevé que celui obtenu dans la région parisienne. Les mesures en Poitou-Charentes sont à faire, mais, compte tenu des différences observées entre les doses dues au rayonnement tellurique lié aux différences géologiques, on peut supposer que les rapports des concentrations moyennes en radon entre Limousin et Poitou-Charentes seront du même ordre de grandeur qu'entre Limousin et région parisienne.

Les deux régions étudiées sont essentiellement à dominante agricole avec quelques villes moyennes de part et d'autre. Bien que nous ne disposions pas d'informations spéciales sur d'éventuelles différences entre ces régions sur les multiples facteurs qui interviennent dans la carcinogénèse, en particulier sur les facteurs socio-économiques, il est vraisemblable que la différence constatée entre les niveaux moyens d'exposition aux rayonnements devrait entraîner au moins une tendance si les faibles doses chroniques avaient l'efficacité que certains leur attribuent. Aussi a-t-il paru intéressant d'entreprendre une étude sur les taux comparatifs de mortalité par cancer dans ces deux régions.

Cette première étude est limitée aux huit années qui vont de 1968 à 1975 et se rapporte à la mortalité par affections malignes tous âges confondus.

\section{DONNEES DE BASE}

Les années 1968 et 1975 sont des années de recensement ; les populations et leur répartition en sexes et classes d'âges (11 classes) sont donc celles qu'indiquent les documents INSEE et INSERM pour ces années. On les trouvera dans le tableau I. Pour les six années intermédiaires, les populations et leur répartition ont été calculées par interpolation exponentielle comme dans une étude antérieure concernant le département de la Manche [3]. La population de référence qui a été choisie est celle adoptée par l'INSERM pour le calcul des taux comparatifs ou standardisés [1] : c'est la population totale (2 sexes) de la France obtenue à partir des résultats du recensement de 1968. Le lecteur en trouvera le détail dans l'article déjà cité [3]. Le nombre de décès par cancer dans l'une et l'autre des régions étudiées 
est donné par les statistiques de causes médicales de décès (statistiques définitives rapportées au lieu de domicile) publiées par l'INSERM [6]. Les tumeurs y sont réparties en 8 classes dont on trouvera dans le tableau II la désignation et les numéros correspondants de la classification internationale des maladies (CIM $8^{e}$ révision) utilisée avant 1979.

\section{TABLEAU I}

Population par sexe et âge pour les régions Limousin et Poitou-Charentes d'après les recensements de 1968 et de 1975

\begin{tabular}{|c|c|c|c|c|}
\hline \multicolumn{5}{|c|}{ RECENSEMENT 1968} \\
\hline & \multicolumn{2}{|c|}{ Limousin } & \multicolumn{2}{|c|}{ Poitou-Charentes } \\
\hline & Masculin & Féminin & Masculin & Féminin \\
\hline 1 an & 4649 & 4458 & 11920 & 11589 \\
\hline $1 \cdot 4$ & 15551 & 14622 & 40060 & 38187 \\
\hline $5 \cdot 14$ & 52824 & 50944 & 131116 & 127552 \\
\hline $15 \cdot 24$ & 53444 & 49548 & 120680 & 113912 \\
\hline $25 \cdot 34$ & 36580 & 35200 & 77200 & 76796 \\
\hline $35 \cdot 44$ & 48360 & 47272 & 94504 & 92404 \\
\hline $45 \cdot 54$ & 40728 & 40076 & 72820 & 74444 \\
\hline $55 \cdot 64$ & 49516 & 52256 & 85736 & 88756 \\
\hline $65-74$ & 36764 & 46248 & 59300 & 74772 \\
\hline $75 \cdot 84$ & 14844 & 28784 & 24932 & 46244 \\
\hline \multirow[t]{2}{*}{85 et +} & 3408 & 8448 & 5884 & 13428 \\
\hline & 356668 & 377856 & 724152 & 758084 \\
\hline
\end{tabular}

\begin{tabular}{|c|c|c|c|c|}
\hline \multicolumn{5}{|c|}{ RECENSEMENT 1975} \\
\hline & \multicolumn{2}{|c|}{ Limousin } & \multicolumn{2}{|c|}{ Poitou-Charentes } \\
\hline & Masculin & Féminin & Masculin & Féminin \\
\hline 1 an & 3943 & 3765 & 10510 & 9963 \\
\hline $1 \cdot 4$ & 15342 & 14545 & 39125 & 37717 \\
\hline $5 \cdot 14$ & 50155 & 48915 & 125785 & 119520 \\
\hline $15 \cdot 24$ & 54340 & 51180 & 127515 & 121095 \\
\hline $25 \cdot 34$ & 45150 & 41445 & 94840 & 91260 \\
\hline $35-44$ & 40780 & 40415 & 84815 & 83565 \\
\hline $45 \cdot 54$ & 49635 & 50475 & 93715 & 94385 \\
\hline $55 \cdot 64$ & 37145 & 38865 & 67085 & 71100 \\
\hline $65 \cdot 74$ & 41790 & 50360 & 69695 & 80855 \\
\hline $75 \cdot 84$ & 17965 & 31210 & 29510 & 51880 \\
\hline \multirow[t]{2}{*}{85 et +} & 3870 & 9995 & 6370 & 16290 \\
\hline & $\overline{360115}$ & $\overline{381170}$ & 748965 & 777630 \\
\hline
\end{tabular}

\section{VALIDITÉ DES DONNÉES}

Comme cela a déjà été exposé dans [3], il y a des incertitudes dans toute étude statistique qui exploite les causes de mortalité indiquées sur les certificats médicaux de décès. Selon l'usage, nous admettrons ici que la validité des données de base se mesure grossièrement au pourcentage 
de "causes spécifiées de décès". Le tableau III en donne les valeurs pour les deux régions étudiées et on peut voir que, de ce point de vue, ces régions présentent une homogénéité satisfaisante.

TABLEAU ॥

Regroupement en 8 classes des tumeurs causes de décès tel qu'il est donné dans les statistiques des causes médicales de décès entre 1968 et 1978 publiées par I'INSERM [6]

\begin{tabular}{|c|c|}
\hline $\begin{array}{c}\text { Numéros de la classification } \\
\text { internationale des maladies } \\
8^{e} \text { révision }\end{array}$ & Causes de décès \\
\hline $140 \cdot 149$ & $\begin{array}{l}\text { Tumeurs malignes de la cavité } \\
\text { buccale et du pharynx }\end{array}$ \\
\hline $150 \cdot 159$ & $\begin{array}{l}\text { Tumeurs malignes de l'appareil } \\
\text { digestif et du péritoine }\end{array}$ \\
\hline $160 \cdot 163$ & $\begin{array}{l}\text { Tumeurs malignes de l'appareil } \\
\text { respiratoire }\end{array}$ \\
\hline $170-174$ & $\begin{array}{l}\text { Tumeurs malignes des os, du tissu } \\
\text { conjonctif, de la peau et du sein }\end{array}$ \\
\hline $180 \cdot 189$ & $\begin{array}{l}\text { Tumeurs malignes des organes } \\
\text { génito-urinaires }\end{array}$ \\
\hline $190-199$ & $\begin{array}{l}\text { Tumeurs malignes de sièges autres } \\
\text { et sans précision }\end{array}$ \\
\hline $204-207$ & Leucémies \\
\hline $200 \cdot 203,208,209$ & $\begin{array}{l}\text { Autres tumeurs des tissus } \\
\text { lymphatiques et hématopoïétiques }\end{array}$ \\
\hline
\end{tabular}

TABLEAU III

Validité des statistiques

Proportion des causes spécifiées de mortalité pour 100 décès (suivant le lieu de domicile)

\begin{tabular}{|c|c|c|}
\hline Années & Limousin & Poitou-Charentes \\
\hline 1968 & 90,5 & 89,4 \\
1969 & 91,8 & 90,9 \\
1970 & 90,6 & 91,2 \\
1971 & 91,9 & 91,4 \\
1972 & 93,2 & 93,5 \\
1973 & 92,6 & 92,9 \\
1974 & 93,5 & 93,5 \\
1975 & 93,1 & 91,6 \\
\hline
\end{tabular}

On n'a pas appliqué la correction préconisée par Breil [2] et par Ledermann [9] dont le principe semble discutable et qui apporterait une précision illusoire devant les incertitudes, difficiles à chiffrer, qui affectent les populations par tranche d'âge obtenues par interpolation pour les années intermédiaires 1969-1974. 


\section{RÉSULTATS ET DISCUSSION}

Les taux comparatifs (nombre de décès pour 100000 habitants et par an) ont été calculés pour les deux régions Limousin et Poitou-Charentes, pour l'un et l'autre sexe, pour chacune des 8 années 1968-1975, pour chacune des 8 classes de tumeurs précisées dans le tableau II.

Le détail des résultats année par année fera l'objet de notes techniques qui seront publiées plus tard. Pour cet article, il a semblé plus significatif de présenter les valeurs moyennes calculées sur les huit années pour les hommes d'une part et pour les femmes d'autre part. Ces moyennes sont présentées dans le tableau IV pour chacune des classes de tumeurs étudiées.

TABLEAU IV

Taux comparatifs de mortalité par tumeurs pour le Limousin et le Poitou-Charentes (pour 100000 habitants et par an)

Moyennes sur les années de 1968 à 1975

\begin{tabular}{|c|c|c|c|c|}
\hline & \multicolumn{2}{|c|}{ Sexe masculin } & \multicolumn{2}{|c|}{ Sexe féminin } \\
\hline & Limousin & Poitou-Charentes & Limousin & Poitou-Charentes \\
\hline Cavité buccale et pharynx & 15,6 & 16,3 & 1,1 & 1,5 \\
\hline Appareil digestif et péritoine & 114,5 & 98,8 & 60,5 & 54 \\
\hline Appareil respiratoire & 52.2 & 53.8 & 6.8 & 6,8 \\
\hline $\begin{array}{l}\text { Os, tissus conjonctifs, } \\
\text { peau et sein }\end{array}$ & 7,5 & 7,7 & 24,2 & 27,8 \\
\hline Organes gènito-urinaires & 42.8 & 45,2 & 24,9 & 28,4 \\
\hline $\begin{array}{l}\text { Tumeurs de sièges autres } \\
\text { et sans précision }\end{array}$ & 31,1 & 27.8 & 23,2 & 21,8 \\
\hline Leucémies & 9,9 & 9,2 & 6 & 5.9 \\
\hline $\begin{array}{l}\text { Autres tumeurs des tissus } \\
\text { lymphatiques et hématopoiétiques }\end{array}$ & 6.9 & 7,8 & 3,4 & 5 \\
\hline Total des affections malignes & 280,5 & 266,6 & 150,1 & 151,2 \\
\hline
\end{tabular}

II est facile de constater que, mis à part le cas des décès par tumeur

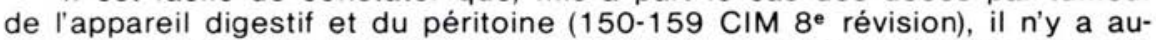
cune différence significative entre les deux régions. L'examen des différences année par année, dont le signe varie de façon aléatoire, confirme pour l'un et l'autre sexe cette conclusion.

Le plus surprenant, par référence à ce que l'on pourrait attendre, est le résultat obtenu pour les tumeurs de l'appareil respiratoire. Le tableau V donne les valeurs pour chacune des huit années étudiées et il est évident qu'il n'y a pas de différence significative entre les deux régions. D'ailleurs, dans une étude en cours de l'INSERM [5] sur la comparaison de la mortalité régionale entre 1975 et 1982 , les taux standardisés (sur la population française de 1968 ) de la mortalité par cancer du poumon ( $N^{\circ} 162$ de CIM $8^{e}$ révision) sont pour le sexe masculin en 1982 : 50,5 pour le Limousin, et 49,3 pour le Poitou-Charentes. Ils sont, l'un et l'autre, classés parmi les taux significativement inférieurs au taux général pour la France entière $(67,9)$. 
TABLEAU $V$

Décès par tumeurs de l'appareil respiratoire Taux comparatifs de 1968 à 1975

\begin{tabular}{|c|c|c|c|c|}
\hline \multirow{2}{*}{ Années } & \multicolumn{2}{|c|}{ Sexe masculin } & \multicolumn{2}{c|}{ Sexe féminin } \\
\cline { 2 - 5 } & Limousin & PoitourCharentes & Limousin & Poitou-Charentes \\
\hline 1968 & 40,8 & 45,7 & 9,4 & 6,4 \\
1969 & 48,6 & 49,3 & 7,7 & 5,3 \\
1970 & 47,4 & 48,4 & 6,1 & 6,6 \\
1971 & 50,4 & 53,5 & 5,1 & 8,4 \\
1972 & 54,8 & 53,1 & 7,3 & 7,3 \\
1973 & 59,5 & 57,8 & 6 & 6,1 \\
1974 & 57,4 & 56,8 & 6,8 & 7 \\
1975 & 58,6 & 65,7 & 5,8 & 7,4 \\
\hline Moyenne & 52,2 & 53,8 & 6,8 & 6,8 \\
\hline
\end{tabular}

Il est donc clair que si la présence du radon à concentration nettement plus élevée dans beaucoup d'habitations du Limousin par rapport à celles du Poitou-Charentes a un effet carcinogène, cet effet est totalement noyé dans l'effet des autres causes liées au mode de vie, à l'environnement, ou aux caractéristiques génétiques des populations.

Parmi les explications possibles, on pourrait imaginer que la consommation de tabac puisse être supérieure en Poitou-Charentes et effacer ainsi la différence qu'il pourrait y avoir entre les deux régions. Un sondage dans les statistiques de vente de tabac et cigarettes de la SEITA [10] montre qu'en 1968 (comme en 1975) la consommation dans le Limousin était légèrement supérieure à celle du Poitou-Charentes (en 1968, 982 cigarettes par habitant contre 875 et 420 grammes de tabac contre 411 ). II est peu probable que dans les années cinquante cette tendance ait été inversée au point d'avoir une influence marquée sur la comparaison entreprise ici des cancers de l'appareil respiratoire pour les années 1968-1975.

Finalement, la seule différence qui ait une certaine signification est celle qui concerne les taux de mortalité par tumeur de l'appareil digestif et du péritoine. Ce fait demande que soit entreprise une étude plus détaillée au niveau des différentes parties de "l'appareil digestif", dénomination générale qui se subdivise en 10 classes à 3 chiffres (150 à 159) dans la $8^{e}$ révision de la $\mathrm{CIM}$. Ce travail sera entrepris dès que les données de base seront disponibles.

Cependant, dans l'étude comparative entre régions déjà citées [5], le Limousin présentait en 1975, pour le sexe masculin, un taux standardisé de mortalité par cirrhose et psychose alcoolique nettement plus élevé que le Poitou-Charentes : 62,8 contre 53,9 . II ne serait donc pas étonnant que la disparité constatée dans le taux de décès par cancer de l'appareil digestif soit, au moins en partie, due à la différence dans la consommation d'alcool.

\section{CONCLUSION}

La poursuite de ces études sur deux populations importantes en nombre et pour lesquelles les différences de dose moyenne $\gamma$ s'établissent à plus 
de 3 rems en 50 ans, ajoutées à des différences beaucoup plus importantes (bien que difficiles à chiffrer en rems) dues à l'irradiation pulmonaire par les descendants à vie courte du radon, devrait apporter des informations utiles dans la recherche sur l'effet carcinogène des faibles doses chroniques. Ce qui semble se dégager de cette première étude c'est que rien ne confirme ici les hypothèses avancées par Stewart, Mancuso et Kneale [8] d'une dose doublante de l'ordre de 15 rems qui, pour les tissus les plus sensibles à l'action carcinogène des rayonnements, devrait entraîner pour le Limousin un excès de 20 à 30 \% de la mortalité par cancer.

\section{BIBLIOGRAPHIE}

[1] BELLANGER F., BERLIE J., BRUNET M., HUCHER M., HATTON F. et MAUJOL L. La mortalité par cancer en l'année 1976 et son évolution depuis 1954. Paris : INSERM, 1979.

[2] BREIL J. Statistique du mouvement de la population. 2e partie : les causes de décès. Année 1943. Paris: INSEE, 1947.

[3] DOUSSET M. et JAMMET H. La mortalité par affections malignes dans le département de la Manche (1962-1980). Radioprotection, 1983, 18 (4) 223-232.

[4] GUEZENGAR J.M., BOUVILLE A. et MADELMONT C. Etude de l'irradiation naturelle en France. Description et premiers résultats du programme du Commissariat à l'énergie atomique. Note CEA-N-2108, 1980.

[5] HATTON F., MAUJOL L. et BOUVIER-COLLE M.H. Mortalité régionale en France, évolution des disparités. INSERM 1984, communication personnelle.

[6] INSERM : Statistiques des causes médicales de décès 1968-1978. Résultats France et par régions (tomes I et II) Paris: INSERM, 1971 à 1982.

[7] JEANMAIRE L. Communication personnelle, 1980.

[8] KNEALE G.W., MANCUSO T.F., STEWART A.M. Hanford radiation study III : a cohort study of the cancer risks from radiation to workers at Hanford (1944-1977 deaths) by the method of regression models in life-tables. Br. J. Ind. Med., 1981, 38, 156-166.

[9] LEDERMANN S. La répartition des décès de cause indéterminée. Rev. Institut Int. Stat., 1956, No 1/3, 47-57.

[10] SEITA. Situtation générale de la consommation des tabacs en 1968 et statistiques départementales annuelles 1975. SEITA, Direction Marketing et ventes recherches commerciales et prévisions. 